\title{
Gestação e obesidade: um problema emergente
}

\author{
Pregnancy and obesity: an emerging problem
}

\author{
Gestación y obesidad: un problema emergente
}

\author{
João Regis I. Carneiro," Fernanda O. Braga, Carolina A. Cabizuca, \\ Raquel C. Abi-Abib, Roberta A. Cobas, Marília B. Gomes
}

\begin{abstract}
Resumo
Diante do crescimento da prevalência de sobrepeso e obesidade na população adulta brasileira, sobretudo nas mulheres em idade fértil, a gestação neste grupo populacional torna-se merecedora de atenção pelas autoridades de saúde no nosso país. Esta condição ainda é pouco estudada, e não há centros de referência para o atendimento das grandes obesas gestantes. Sabemos que grandes obesas têm chance de iniciar a gestação com a saúde comprometida por uma variedade de doenças crônicas, entre as quais podemos citar: hipertensão arterial, diabetes tipo 2, asma brônquica e esteatose hepática. Alguns estudos já mostraram aumento de risco para o binômio mãe/ feto para além do período fetal. A gestação deve ser cuidadosamente planejada, uma vez que os métodos contraceptivos podem sofrer perda de sua eficácia pelo excesso ponderal nestas mulheres e em especial nas que se submeteram à cirurgia bariátrica. O manejo do período pré-natal requer atenção diferenciada, visando controlar o ganho ponderal materno e minimizar as complicações fetais já conhecidas, como macrossomia, anomalias e deficiências nutricionais. Os achados desta revisão demonstram que o excesso de peso na gestação constitui fator de risco para a saúde da mãe e do concepto. A pandemia de obesidade, que agora atinge países em desenvolvimento, afeta de forma especial mulheres em idade fértil. A gestação em grandes obesas gera necessidade aumentada de exames pré-natais, maior número de partos cesáreos, aumento de infecções em sítio cirúrgico, maior tempo de internação - o que leva a grande impacto econômico para o sistema de saúde pública. São necessários mais estudos e investimentos em capacitação de equipes de saúde e materiais específicos para atender estas pacientes.
\end{abstract}

Descritores: Obesidade mórbida; Obesidade; Cirurgia bariátrica; Gravidez.

\begin{abstract}
Because of the increasing prevalence of overweight and obesity in the Brazilian adult population, particularly in women in reproductive age, pregnancy in this population deserves attention by health authorities. This condition is still poorly understood, and there are no reference centers for maternal super-obesity care. Obese women are at an increased risk of chronic comorbid conditions, such as hypertension, type 2 diabetes, asthma and nonalcoholic fat liver disease. Some studies show that the increased risk to both mother and fetus extends beyond intrauterine period. Gestation should be planned, as obesity may impair the efficacy of contraceptive methods, especially in those women who underwent bariatric surgery. Prenatal care requires special attention so as to control maternal weight gain and to minimize known fetal complications such as macrosomia, anomalies and nutritional deficiencies. This review demonstrates that excessive weight during pregnancy is an important risk factor for the health of both mother and fetus. The obesity pandemic has come to afflict developing countries and affects women in reproductive age in a particular way. Pregnancy in morbid obese women requires a greater number of prenatal exams, demands more frequent cesarean labors, increases the incidence of surgical infections, demands longer hospitalization - which leads to greater economical impact on the public health system. Further studies and investment in training for health personnel are required to assist these patients.
\end{abstract}

Keywords: Obesity; Obesity, Morbid; Bariatric surgery; Pregnancy.

Revista HUPE, Rio de Janeiro, 2014;13(3):17-24

Recebido: 10/03/2014 | Revisado: 02/07/2014 | Aprovado: 28/07/2014

doi: 10.12957/rhupe.2014.12130
"Endereço para correspondência: Serviço de Diabetes, Hospital Universitário Pedro Ernesto. Boulevard 28 de Setembro, 77, 3ㅇ andar. Rio de Janeiro, RJ. CEP: 20551-030. E-mail: endoregis@uol.com.br 


\section{Resumen}

Frente al crecimiento de la prevalencia de sobrepeso y obesidad en la población adulta brasilera, sobre todo en las mujeres en edad fértil, la gestación en este grupo poblacional se vuelve merecedora de atención por parte de las autoridades de salud en nuestro país. Esta condición todavía es poco estudiada, y no hay centros de referencia para la atención de las grandes obesas en gestación. Sabemos que éstas tienen predisposición a iniciar una gestación con la salud comprometida por una variedad de enfermedades crónicas, entre las cuales podemos citar: hipertensión arterial, diabetes tipo 2, asma bronquial, esteatosis hepática. Algunos estudios ya mostraron aumento de riesgo para el binomio madre/feto más allá del periodo fetal. La gestación debe ser planeada, una vez que los métodos contraceptivos pueden sufrir pérdida de su eficacia por el exceso ponderal en estas mujeres; en especial en las que se sometieron a cirugía bariátrica. El manejo del periodo prenatal requiere atención diferenciada, apuntando a controlar la ganancia ponderal materna y minimizar las complicaciones fetales ya conocidas, como macrosomías, anomalías y deficiencias nutricionales. Los hallazgos de esta revisión demuestran que el exceso de peso en la gestación constituye un factor de riesgo para la salud de la madre y del producto. La pandemia de obesidad, que ahora alcanza a países en desarrollo, afecta de forma especial a mujeres en edad fértil. La gestación en grandes obesas genera una mayor necesidad de exámenes prenatales, un mayor número de partos por cesárea, aumento de infecciones en sitio quirúrgico, mayor tiempo de internación - lo que lleva a un gran impacto económico para el sistema de salud pública. Son necesarios más estudios e inversiones en capacitación de equipos de salud y materiales específicos para atender a éstas pacientes.

Palabras clave: Obesidad mórbida; Obesidad; Cirugía bariátrica; Gravidez.

\section{Obesidade - conceitos}

Nos últimos anos, a obesidade vem adquirindo espaço na mídia e no meio científico. Antes considerada um distúrbio de pouca importância clínica - a própria comunidade científica considerava a obesidade um distúrbio relacionado a maus hábitos e pouca determinação -, ela afetaria então um indivíduo sem força de vontade e totalmente responsável pela própria condição clínica. Hoje, porém, sabe-se que o excesso de peso é um distúrbio de origem multifatorial. ${ }^{1}$ Em sua gênese, estão fatores genéticos, culturais, socioeconômicos, metabólicos e psíquicos. $\mathrm{O}$ excesso ponderal é, portanto, uma condição crônica extremamente difícil de tratar, sobretudo se considerarmos resultados mantidos após longos períodos de observação. ${ }^{2}$
O conceito de obeso corresponde àqueles que apresentam excesso de tecido adiposo em sua composição corporal. O índice de massa corporal (IMC em kg/m²), apesar de impreciso, constitui a maneira mais comumente utilizada em todo o mundo para se diagnosticar o excesso de peso e os diferentes graus de obesidade. Indivíduos com IMC igual ou superior a $25 \mathrm{~kg} / \mathrm{m}^{2}$ são denominados com excesso de peso. A partir de 30 $\mathrm{kg} / \mathrm{m}^{2}$, há obesidade de grau I; a partir de $35 \mathrm{~kg} /$ $\mathrm{m}^{2}$, obesidade de grau II, e, a partir de $40 \mathrm{~kg} / \mathrm{m}^{2}$, configura-se um quadro de obesidade mórbida ou de obesidade grau III. O crescimento da epidemia de obesidade mórbida justificou a necessidade da criação de uma nova classificação. Aqueles com IMCs iguais ou superiores a 50 e $60 \mathrm{~kg} / \mathrm{m}^{2}$ são considerados, respectivamente, superobesos e supersuperobesos. ${ }^{3}$ (Tabela 1)

Tabela 1. Classificação de sobrepeso e obesidade pelo IMC.

\begin{tabular}{c|c}
\hline IMC $\left(\mathbf{k g} / \mathbf{~ m}^{\mathbf{2}}\right)$ & Classificação \\
\hline $18,5-24,99$ & Normal \\
\hline $25-29,99$ & Sobrepeso \\
\hline $30-34,99$ & Obesidade grau I \\
\hline $35-39,99$ & Obesidade grau II \\
\hline $40-49,99$ & Obesidade grau III (mórbida) \\
\hline $50-59,99$ & Superobesidade \\
\hline$\geq 60$ & Super superobesidade \\
\hline
\end{tabular}


Seguindo esta classificação, tem se observado nas últimas décadas um importante aumento na prevalência do excesso de peso e da obesidade em nosso país. ${ }^{4} \mathrm{O}$ crescimento econômico do país trouxe inúmeros benefícios, entre os quais a redução da mortalidade infantil e da desnutrição. Entretanto, as mudanças no comportamento social e alimentar da nossa população justificam plenamente esta variação antropométrica que tanto nos preocupa. Parodiando o economista americano John Kenneth Galbraith, podemos dizer que, no Brasil de hoje, morrem mais pessoas por excesso que por falta de alimentos.

De fato, os obesos têm sua expectativa de vida abreviada por sua condição clínico-metabólica. ${ }^{5}$ São mais propensos ao desenvolvimento de hipertensão arterial, resistência insulínica e diabetes do tipo 2 (DM 2), ${ }^{6}$ assim como de distúrbios respiratórios ${ }^{7}$ e ortopédicos. ${ }^{8} \mathrm{O}$ impacto do excesso de peso nos níveis de glicemia plasmática já foi exaustivamente demonstrado. A obesidade interfere sobremaneira na fisiopatologia do diabetes mellitus do tipo 2 (DM 2). ${ }^{5}$

A distribuição da gordura corporal merece atenção especial, uma vez que a chamada obesidade androide (distribuição "central” da gordura) guarda maior relação com o desenvolvimento de diabetes, hipertrigliceridemia, redução dos níveis plasmáticos de colesterol HDL, hipertensão e doença cardiovascular. ${ }^{9}$

A obesidade associa-se também ao aumento do risco de neoplasia de endométrio ${ }^{10}$ e mama. ${ }^{11}$
É preocupante a dificuldade que grandes obesas encontram, em realizar exames de rastreio para estas condições em função das limitações técnicas que a maioria absoluta dos equipamentos disponíveis no sistema de saúde brasileiro apresenta, a partir de determinado limite de peso.

\section{Obesidade e fertilidade}

A associação entre obesidade e alterações no ciclo menstrual foi descrita em 1952 por Rogers e Mitchell. ${ }^{12}$ Neste estudo, $43 \%$ das mulheres com ciclos menstruais irregulares eram obesas, enquanto apenas $13 \%$ daquelas que menstruavam normalmente estavam acima do peso.

Na medicina antiga, Hipócrates já notava que mulheres obesas tinham mais dificuldade em conceber, e isto era atribuido à compressão que a gordura em excesso exerceria sobre o útero e as trompas. ${ }^{13}$

Paradoxalmente, diversas esculturas da era antiga retratam mulheres obesas, cujas cabeças e membros minúsculos contrastam com as generosas proporções dedicadas ao abdome e à mama. De fato, é possível que, no passado, o excesso de peso tenha sido relacionado com a fertilidade, a prosperidade e a fortuna.

Entretanto, em mulheres obesas ou com sobrepeso diagnosticadas com síndrome de ovários policísticos, a perda de peso por dieta e atividade física é capaz de promover alterações morfológicas ovarianas, com redução do número de micro-

Tabela 2. Contracepção em mulheres obesas.

\begin{tabular}{c|c|c}
\hline \multicolumn{2}{c}{$\begin{array}{c}\text { Critério de Elegibilidade Médica (CEM) para escolha do método contraceptivo em mulheres obesas } \\
\text { Adaptado de Jeniffer A Robinson \& Anne E Burke - Women's Health (2013) 9 (5), 453-466 }\end{array}$} \\
\hline Método Contraceptivo & Categoria CEM & Definição \\
\hline D.I.U (contracepção intrauterina) & 1 & Categoria 1: sem restrição ao uso \\
\hline Implante de progestágeno & 1 & Categoria 1: sem restrição ao uso \\
\hline Acetato de medroxiprogesterona (depósito) & 1 & Categoria 1: sem restrição ao uso \\
\hline “Minipílulas" (progestágeno isolado) & 1 & Categoria 1: sem restrição ao uso \\
\hline Pílulas contraceptivas de emergência (pílula do \\
dia seguinte)
\end{tabular}


cistos, reestabelecendo a regularidade dos ciclos ovulatórios e a fertilidade. ${ }^{14}$ Ressalta-se ainda a relação inversa entre aumento do IMC e sucesso terapêutico de técnicas de fertilização in vitro. ${ }^{15}$

\section{Anticoncepção em mulheres obesas}

Algumas questões devem ser consideradas antes da escolha da melhor opção para anticoncepção em mulheres obesas. O uso de métodos contraceptivos uterinos (DIU) parece ser uma opção adequada para grandes obesas. Implantes de etonogestrel parecem ter eficácia reduzida em mulheres com IMCs muito elevados, sendo sugerida troca do dispositivo antes dos 3 anos de permanência, tempo limite estabelecido pelo fabricante. Diversos estudos sugerem discreta redução na eficácia de anticoncepcionais combinados estrogênio/progestágeno por via oral. Além de redução na adesão terapêutica observada em mulheres obesas, o aumento do IMC parece potencializar o impacto da falta do uso do comprimido na redução da eficácia deste composto. Da mesma forma, a pílula do dia seguinte, à base de levonogestrel, pode ter eficácia reduzida em grandes obesas.

Em resumo, apesar de haver uma discreta redução na eficácia com grandes aumentos do IMC, os diversos métodos de anticoncepção disponíveis no mercado podem ser utilizados com segurança por mulheres obesas. Atenção especial deve ser dedicada ao aumento do risco de desenvolvimento de trombose venosa profunda em usuárias de anticoncepcionais hormonais combinados. Para as portadoras de obesidade grau III são necessários mais estudos para se estabelecer a segurança e eficácia dos diversos métodos de anticoncepção disponíveis no mercado. ${ }^{16}$ (Tabela 2)

\section{Gestação e obesidade}

Considerando o crescimento da prevalência do excesso de peso e da obesidade na população adulta brasileira - atualmente aproximadamente $50 \%$ das mulheres brasileiras em idade fértil estão acima do peso -, não é difícil considerar que, apesar de ainda pouco estudada, a gestação em mulheres obesas surge como uma questão que merece toda a atenção por parte das autoridades de saúde do nosso país. Torna-se, ainda, necessária a criação de centros de referência para o atendimento de grandes obesas gestantes. Um recente estudo desenvolvido pelo Serviço de Diabetes do HUPE e apresentado no último Congresso Brasileiro de Diabetes ${ }^{17}$ chamou atenção para a significativa prevalência de obesas grau III com diabetes gestacional (DMG) acompanhadas em conjunto pelos Serviços de Obstetrícia e Diabetes da UERJ entre 2011 e 2012. Das 43 pacientes estudadas, 58,13\% eram obesas, sendo que em $44 \%$ destas o IMC no momento da concepção era superior a $40 \mathrm{~kg} / \mathrm{m}^{2}$. A obesidade mórbida constitui, de fato, um problema ainda mais complexo. Nas últimas três décadas houve um incremento de $285 \%$ na prevalência de obesidade grau III na população adulta do sudeste brasileiro. ${ }^{18}$ Estima-se que no estado do Rio de Janeiro, em torno de 120 mil pessoas possam ser, hoje, classificadas como portadores de obesidade mórbida. Se considerarmos que aproximadamente metade destas pessoas pode ser constituída de mulheres em idade fértil, não seria difícil imaginar que qualquer esforço no sentido de otimizar o atendimento às gestantes obesas deve ser valorizado e estimulado, mesmo considerando o impacto negativo que o excesso de peso pode causar na fertilidade. Grandes obesas têm uma chance significativa de iniciar a gestação com a saúde comprometida por uma variedade de problemas de saúde crônicos.

A hipertensão arterial sistêmica, (HAS) há muitos anos, é o fator de maior impacto na avaliação do risco de morte materna durante a gestação. A associação entre obesidade, HAS e doença cardiovascular já foi claramente demonstrada. $\mathrm{O}$ excesso de tecido adiposo pode promover ativação do sistema renina-angiotensina-aldosterona, aumento do tônus simpático, além de estar associado à produção de fatores procoagulação e disfunção endotelial. ${ }^{19}$ A síndrome da apneia obstrutiva do sono, bastante prevalente em grandes obesas, pode contribuir para o aumento da pressão arterial e para o desenvolvimento de arritmias através de uma ativação simpática ainda maior. ${ }^{20}$

A Síndrome de Resistência insulínica relaciona-se de maneira inequívoca com a obesidade e tem papel fundamental na fisiopatogênese do DM2, doença bastante prevalente em grandes obesas. Outrossim, a gestação é condição acompanhada de grande redução fisiológica da ação da insulina em seus tecidos alvo. Destaca-se o papel da placenta como sítio de produção de ci- 
tocinas inflamatórias que interferem na resposta tissular à ação da insulina. ${ }^{19}$ Mulheres obesas têm, assim, risco aumentado de desenvolver diabetes gestacional, condição de agravo à saúde da mãe e do concepto.

A esteatose hepática, presente em mais de 90\% das pacientes com obesidade grau III, pode tornar mais grave a evolução de doenças hepáticas associadas à gestação. ${ }^{21}$ Grandes obesas podem apresentar disfunção renal independente da presença de hipertensão ou diabetes mellitus. A recém-descrita "nefropatia da obesidade" está associada à síndrome da apneia obstrutiva do sono e pode ser revertida com a perda de peso. ${ }^{22}$ Encontra-se associação entre obesidade mórbida e asma brônquica, síndrome da apneia obstrutiva do sono, infecções cutâneas de repetição e distúrbios osteomusculares.

Mesmo aquelas que iniciam a gestação livres destas condições, têm risco aumentado de desenvolver pré-eclâmpsia e diabetes gestacional. O aumento do IMC está relacionado com o aumento na indicação de cesárea, infecções na ferida cirúrgica, hemorragias e fetos macrossômicos e com anomalias. ${ }^{23}$ Ressalta-se ainda a possibilidade de filhos de mães obesas apresentarem, já na adolescência, distúrbios relacionados à síndrome metabólica. ${ }^{21}$

\section{Recomendações de ganho de peso na gestação}

Em condições normais, aproximadamente $60 \%$ do total do ganho de peso durante a gestação pode ser atribuído a fatores maternos, que correspondem à expansão do volume sanguíneo e do líquido extracelular, ao aumento das mamas

e do útero e, principalmente, ao aumento dos depósitos de tecido adiposo da mãe. O feto contribui com o líquido amniótico, a placenta e seu próprio peso. ${ }^{19}$ Recentemente, estabeleceu-se recomendação para ganho de peso baseada no estado nutricional da mãe antes da concepção. Embora acertada, ainda deve passar por revisões no futuro, sobretudo por não contemplar a emergente população de gestantes portadoras de obesidade grau III que procuram as maternidades em todo o mundo. (Tabela 3)

Há na literatura evidências de que um ganho total de peso inferior a $5 \mathrm{~kg}$ está relacionado a uma menor mortalidade perinatal e recém-natos com peso mais apropriado naquelas que iniciam a gestação com elevados índices de massa corpórea. ${ }^{24}$ Sendo assim, a gestação poderia ser um momento oportuno para se investir no controle do peso. Grandes obesas que planejam engravidar deveriam se sensibilizar no sentido de iniciar um projeto visando perda ponderal e melhora de eventuais comorbidades relacionadas à obesidade antes da concepção.

Não menos importante é o impacto econômico que o excesso de peso pode causar no curso da gestação. Este fator torna mais prováveis a realização de um número maior de exames e consultas no período pré-natal, a utilização de medicamentos para controle da pressão e do diabetes gestacional, o aumento de indicação de cesárea e a incidência de complicações que podem prolongar o período de internação hospitalar no peri/pós parto. ${ }^{25}$

\section{Gestação no pós-operatório de cirurgia bariátrica}

Tabela 3. Ganho de peso na gestação x IMC materno.

\begin{tabular}{c|c|c|c}
\hline $\begin{array}{c}\text { IMC pré gestação } \\
\left(\mathbf{k g} / \mathbf{m}^{2}\right)\end{array}$ & $\begin{array}{c}\text { Ganho total no } \\
\text { 10 trimestre }(\mathbf{k g})\end{array}$ & $\begin{array}{c}\text { Ganho semanal no } \\
\text { 20 e 3 trimestres }(\mathbf{k g})\end{array}$ & $\begin{array}{c}\text { Ganho total na } \\
\text { gestação (kg) }\end{array}$ \\
\hline$<18,5$ & 2,3 & 0,5 & $12,5-18,0$ \\
\hline de 18,5 a 24,9 & 1,6 & 0,4 & $11,5-16,0$ \\
\hline de 25 a 29,9 & 0,9 & 0,4 & $7,0-11,5$ \\
\hline
\end{tabular}




\section{Anticoncepção}

A maioria das pessoas submetidas à cirurgia bariátrica nos dias de hoje é composta por muIheres, boa parte delas em idade fértil. A perda de peso decorrente da cirurgia pode restabelecer ciclos ovulatórios, sobretudo naquelas que apresentavam síndrome de resistência à insulina e/ou síndrome de ovários policísticos no pré-operatório. Em função do impacto que algumas técnicas cirúrgicas podem exercer na absorção e metabolismo de diversas substâncias, medicamentos etc., inclusive anticoncepcionais orais, muita atenção deve ser dedicada ao acompanhamento destas mulheres no período pós-operatório, sobretudo pela recomendação expressa de não engravidar antes de decorridos pelo menos 18 meses da cirurgia. $\mathrm{O}$ efeito de anticoncepcionais utilizados pela via oral parece inalterado nas técnicas cirúrgicas classificadas como restritivas (banda gástrica e gastroplastia em manga ou sleeve). Nas técnicas que possuem componente malabsortivo (by pass gastro jejunal com Y de Roux, derivação biliopancreática de Scopinaro), a eficácia destes compostos pode ser bastante reduzida. ${ }^{26}$ (Tabela 4)

\section{Considerações sobre acompanhamento} pré-natal

Gestantes no pós-operatório de cirurgia bariátrica devem ser acompanhadas com suporte de equipe multidisciplinar especializada, preferencialmente por aquela que participou da fase de preparo para a cirurgia. Neste preparo, devem ser fornecidas recomendações expressas para que se evite a gestação durante o período de perda rápida de peso que compreende os primeiros 12-18 meses de pós-operatório.

Embora a cirurgia bariátrica reduza significativamente o risco de desenvolvimento de diabetes gestacional, hipertensão, pré-eclâmpsia, macrossomia e indicação de cesárea ${ }^{19,27}$, atenção especial deve ser dedicada à monitorização de reservas e reposição de nutrientes, principalmente vitamina B12, ácido fólico, vitamina A, ferro, cálcio, vitamina D e tiamina. ${ }^{19,27}$ Nas pacientes que apresentam vômito, torna-se necessária a reposição parenteral de tiamina para prevenção da síndrome de Wernicke. A reposição de ácido fólico também pode ser importante para reduzir o risco de homocisteinemia e eventos vasculares

Tabela 4. Contracepção no pós-operatório de cirurgia bariátrica.

Critério de Elegibilidade Médica (CEM) para escolha de métodos contraceptivos em mulheres após cirurgia bariátrica - Adaptado de Jeniffer A Robinson \& Anne E Burke, 2013

\begin{tabular}{|c|c|c|c|}
\hline Tipo & CEM após cirurgia restritiva & $\begin{array}{l}\text { CEM após cirurgia } \\
\text { disabsortiva }\end{array}$ & Definição \\
\hline $\begin{array}{c}\text { D.I.U } \\
\text { (contracepção intrauterina) }\end{array}$ & 1 & 1 & $\begin{array}{c}\text { Categoria 1: } \\
\text { sem restrição ao uso }\end{array}$ \\
\hline Implante de progestágeno & 1 & 1 & $\begin{array}{c}\text { Categoria 1: } \\
\text { sem restrição ao uso }\end{array}$ \\
\hline $\begin{array}{l}\text { Acetato de medroxiprogesterona } \\
\text { (depósito) }\end{array}$ & 1 & 1 & $\begin{array}{c}\text { Categoria 1: } \\
\text { sem restrição ao uso }\end{array}$ \\
\hline $\begin{array}{c}\text { "Minipílulas" } \\
\text { (progestágeno isolado) }\end{array}$ & 1 & 1 & $\begin{array}{c}\text { Categoria 1: } \\
\text { sem restrição ao uso }\end{array}$ \\
\hline $\begin{array}{l}\text { Pílulas contraceptivas de } \\
\text { emergência (pílula do dia } \\
\text { seguinte) }\end{array}$ & 1 & 3 & $\begin{array}{l}\text { Categoria 3: riscos teóricos } \\
\text { ou comprovados, frequente- } \\
\text { mente, se sobrepõem às } \\
\text { vantagens do método }\end{array}$ \\
\hline $\begin{array}{c}\text { Pílula combinada, adesivo, } \\
\text { anel vaginal }\end{array}$ & 1 & 3 & $\begin{array}{l}\text { Categoria 3: riscos teóricos } \\
\text { ou comprovados, frequente- } \\
\text { mente, se sobrepõem às } \\
\text { vantagens do método }\end{array}$ \\
\hline
\end{tabular}


em gestantes submetidas à cirurgia bariátrica. Os obstetras precisam também ficar atentos ao risco de hérnias internas e isquemia de intestino delgado em gestantes submetidas a técnicas de by pass. ${ }^{27}$

\section{Conclusões}

A obesidade é um problema de saúde pública que deve receber atenção especial por parte das autoridades de saúde. A verdadeira pandemia que vem se instalando não respeita fronteiras: é realidade tanto em países desenvolvidos, como naqueles ditos em desenvolvimento. Como consequência, o número de grandes obesas gestantes que procuram os serviços de obstetrícia em nosso país vem crescendo, tornando necessários investimentos em materiais específicos para atendê-las, como macas, instrumentos cirúrgicos e cadeiras.

O excesso de peso na gestação constitui fator de risco para a saúde da mãe e do concepto, e não menos importante é o impacto econômico do aumento da realização de exames e procedimentos e da utilização de medicamentos. ${ }^{25}$ Com o crescente número de realização das diversas técnicas cirúrgicas bariátricas, é importante também que o obstetra tenha conhecimento do impacto destes procedimentos na terapia de anticoncepção e na condução da gestação.

\section{Referências}

1. Hill JO, Wyatt HR, Melanson EL. Genetic and environmental contributions to obesity. Med Clin North Am, 2000;84(2):333-46.

2. Ayyad C, Andersen T. Long-term efficacy of dietary treatment of obesity: a systematic review of studies published between 1931 and 1999. Obes Rev, 2000;1(2):113-9.

3. Renquist K. Obesity classification. Obes Surg, 1997;7(6):523.

4. Ministério do Planejamento, Orçamento e Gestão, Instituto Brasileiro de Geografia e Estatística-IBGE, Diretoria de Pesquisas, Coordenação de Trabalho e Rendimento. Pesquisa de Orçamentos Familiares 2008-2009 Antropometria e Estado Nutricional de Crianças, Adolescentes e Adultos no Brasil. Rio de Janeiro: Instituto Brasileiro de Geografia e Estatística-IBGE, 2010.

5. Stewart ST, Cutler DM, Rosen AB. Forecasting the Effects of Obesity and Smoking on US Life Expectancy. N Eng J Med, 2009;361(23):2252-60.
6. DeFronzo RA, Ferrannini E. Insulin Resistance: A Multifaceted Syndrome Responsible for NIDDM, Obesity, Hypertension, Dyslipidemia and Atherosclerotic Cardiovascular Disease. Diabetes Care, 1991;14(3):173-94.

7. Weiss ST, Shore S. Obesity and Asthma. Directions for Research. Am J Respir Crit Care Med, 2004;169(8):963-8.

8. Vincent HK, Seay AN, Montero C, Vincent KR. Outpatient Rehabilitation Outcomes in Obese Patients with Orthopedic conditions. Eur J Phys Rehabil Med, 2013;49(3),419-29.

9. Samson SL, Garber AJ. Metabolic Syndrome. Endocrinol Metab Clin North Am, 2014;43(1):1-23.

10. Schmandt RE, Iglesias DA, Co NN, Lu KH. Understanding Obesity and Endometrial Cancer Risk: Opportunities for Prevention. Am J Obstet Gynecol, 2011;205(6):518-25.

11. Calle EE, Kaaks R. Overweight, Obesity and Cancer: Epidemiological Evidence and Proposed Mechanisms. Nature Reviews Cancer, 2004;4(8):579-91.

12. Rogers J, Mitchell GM Jr. The Relationhsip of Obesity to Menstrual Disturbances. N Eng J Med, 1952;247:53-5.

13. On Airs, Waters and Places, Hippocrates, writen 400 BCE. Translated by Francis Adams. Disponível em: http://classics.mit.edu/Hippocrates/airwatpl.html.

14. Crosignani PG, Colombo M, Vegetti W, Somigliana E, Gessati A, Ragni G. Overweight And Obese Anovulatory Patients With Polycystic Ovaries: Parallel Improvements In Anthropometric Indices, Ovarian Physiology And Fertility Rate Induced By Diet. Hum Reprod, 2003;18(9):1928-32.

15. Loveland JB, McClamrock HD, Malinow AM, Sharara Fl. Increased Body Mass Index Has a Deleterious Effect on In Vitro Fertilization Outcome. J Assist Reprod Genet, 2001;18(7):382-6.

16. Robinson JA, Burke AE. Obesity and Hormonal Contraceptive efficacy. Women's Health, 2013;9(5):453-66

17. Braga FO, Carneiro JR, Torres AV, Cobas RA, Duarte JL, Garabini P, et al. Perfil e Evolução das Gestações em Mulheres com Diagnóstico de Diabetes Gestacional em um Hospital Universitário. Arq Bras Endocrinol Metab, 2013; 57(6 ):S371-S372.

18. Santos LM, de Oliveira, Peters LR, Conde WL. Trends in Morbid Obesity and in Bariatric Surgeries Covered by the Brazilian Public Health System. Obes Surg, 2010;20(7):943-8.

19. Rasmussen KM, Yaktine AL. Weight Gain During Pregnancy: Reexamining the Guidelines. The National Academies Press, 2009. 234 p. 
20. Narkiewics K, Somers VK. The Sympathetic Nervous System and Obstructive Sleep Apnea: Implications for Hypertension. J Hypertens, 1997;15(12):1613-9.

21. Catalano PM. Management of Obesity in Pregnancy. Obstet Gynecol, 2007;109(2):419-33.

22. Bayliss G, Weinrauch LA, D’Elia JA. Pathophysiology of Obesity-Related Renal Dysfunction Contributes to Diabetic Nephropaty. Curr Diab Rep, 2012;12(4):440-6. http://dx.doi.org/10.1007/s11892-012-0288-1

23. Marshal NE, Guild C, Cheng YW, Caughey AB, Halloran DR. Maternal Superobesity and Perinatal Outcomes. Am J Obstet Gynecol, 2012;206(5):417.e1-6. http://dx.doi.org/10.1016/j.ajog.2012.02.037

24. Ásbjörnsdóttir B, Rasmussen SS, Kelstrup L, Damm P, Mathiesen ER. Impact of Restricted Maternal Weight Gain on Fetal Growth and Perinatal Morbidity in Obese Women With Type 2 Diabetes. Diabetes Care, 2013;36(5):1102-6. http://dx.doi.org/10.2337/dc12-1232

25. Chu SY, Bachman DJ, Callaghan WM, Witlock EP, Dietz PM, Berg CJ, et al. Association between Obesity during Pregnancy and Increased Use of Health Care. N Engl J Med, 2008;358(14):1444-53. http://dx.doi.org/10.1056/NEJMoa0706786

26. Robinson JA, Burke AE. Obesity and Hormonal Contraceptive efficacy. Women's Health, 2013;9(5):453-66.

27. Mechanick Jl, Kushner RF, Sugerman HJ, GonzalezCampoy M, Collazo-Clavell MLC, Guven S, et al. American Association of Clinical Endocrinologists, The Obesity Society, and American Society for Metabolic \& Bariatric Surgery Medical Guidelines for Clinical Practice for the Perioperative Nutritional, Metabolic, and Nonsurgical Support of the Bariatric Surgery Patient. Surg Obes Relat Dis, 2008;4:S109-S184.

\section{João Regis I. Carneiro}

Disciplina de Diabetes e Metabologia. Departamento de Medicina Interna. Faculdade de Ciências Médicas. Universidade Estadual do Rio de Janeiro. Rio de Janeiro, RJ, Brasil.

\section{Fernanda O. Braga}

Disciplina de Diabetes e Metabologia. Departamento de Medicina Interna. Faculdade de Ciências Médicas. Universidade Estadual do Rio de Janeiro. Rio de Janeiro, RJ, Brasil.

\section{Carolina A. Cabizuca}

Disciplina de Diabetes e Metabologia. Departamento de Medicina Interna. Faculdade de Ciências Médicas. Universidade Estadual do Rio de Janeiro. Rio de Janeiro, RJ, Brasil.

\section{Raquel C. Abi-Abib}

Disciplina de Diabetes e Metabologia. Departamento de Medicina Interna. Faculdade de Ciências Médicas. Universidade Estadual do Rio de Janeiro. Rio de Janeiro, RJ, Brasil.

\section{Roberta A. Cobas}

Disciplina de Diabetes e Metabologia. Departamento de Medicina Interna. Faculdade de Ciências Médicas. Universidade Estadual do Rio de Janeiro. Rio de Janeiro, RJ, Brasil.

\section{Marília B. Gomes}

Disciplina de Diabetes e Metabologia. Departamento de Medicina Interna. Faculdade de Ciências Médicas. Universidade Estadual do Rio de Janeiro. Rio de Janeiro, RJ, Brasil. 